\title{
Epidemiological Profile of Pressure Ulcers in the Emergency Department of the University Hospital Center of Hospital du Mali
}

\author{
Bakary Dembélé ${ }^{1, *}$, Abdoulaye Zié Kone², Yacouba Lazare Diallo ${ }^{3}$, Sow Djénéba Sylla ${ }^{3}$, \\ Mody Traore $^{4}$, Mamadou Hema Ouattara ${ }^{5}$, Ousmane Attaher ${ }^{1}$, Boubacar Togola ${ }^{6}$ \\ ${ }^{1}$ General Directorate of the Hospital du Mali, Bamako, Mali \\ ${ }^{2}$ Ségou Regional Directorate of Veterinary Services, Bamako, Mali \\ ${ }^{3}$ Department of Medicine of the Hospital du Mali, Bamako, Mali \\ ${ }^{4}$ Entrance Office Service, Hospital du Mali, Bamako, Mali \\ ${ }^{5}$ Intensive Care Unit of the Hospital du Mali, Bamako, Mali \\ ${ }^{6}$ Emergency Department of the Hospital du Mali, Bamako, Mali
}

Email address:

ntodembe@yahoo.fr (B. Dembélé)

${ }^{*}$ Corresponding author

\section{To cite this article:}

Bakary Dembélé, Abdoulaye Zié Kone, Yacouba Lazare Diallo, Sow Djénéba Sylla, Mody Traore, Mamadou Hema Ouattara, Ousmane Attaher, Boubacar Togola. Epidemiological Profile of Pressure Ulcers in the Emergency Department of the University Hospital Center of Hospital du Mali. Clinical Medicine Research. Vol. 10, No. 4, 2021, pp. 129-132. doi: 10.11648/j.cmr.20211004.14

Received: July 5, 2021; Accepted: July 16, 2021; Published: July 24, 2021

\begin{abstract}
Introduction: Pressure ulcers are skin lesions of ischemic origin linked to compression of the soft tissues between a hard surface and the bony protrusions. In 2016, 5.17\% of patients developed preventable pressure ulcers in hospital. From this study, the Hôpital du Mali reinforced the care teams and involved carers in pressure ulcer prevention actions. The objective of this study was to assess the prevalence of pressure ulcers as well as prevention actions and their management in the emergency department. Method: This was a cross-sectional descriptive study from January 1 to December 31, 2020, based on the observation of the onset of pressure ulcers in patients. We included all patients hospitalized at the short-stay unit level. Results: of the 305 patients hospitalized during the period, 15 developed bedsores, or $4.9 \%$ prevalence rate. The sex ratio was 1.2. The average age of our series is $52 \pm 14$ years old, ranging between 21 and 87 years old. Seventy percent $(70 \%)$ of pressure ulcers were discovered at stage I. Pressure ulcers located in the inter-gluteal folds were the most frequent with $90 \%$. Conclusion: this study has enabled us to understand that the prevalence of pressure ulcers has decreased from 5.17\% to $4.9 \%$ over the past four years. It encourages us to deepen the examination of the clinical, therapeutic and progressive peculiarities of this disease which is a credible indicator of the quality of care in a hospital structure.
\end{abstract}

Keywords: Epidemiological Profile, Prevalence, Pressure Ulcer, Hôpital du Mali

\section{Introduction}

Background Pressure ulcers are skin lesions of ischemic origin associated with compression of soft tissues between a hard surface and bony protrusions. It is a deep-rooted, inwardly-shaped, conical-shaped wound of multifactorial origin [1]. Local pressure and loss of mobility play a major role in the occurrence of these lesions. [2]. The appearance and development of pressure ulcers in a patient is an important indicator in the appreciation of the quality of care in a hospital structure. This is a significant factor that contributes to the length of a patient's hospital stay. [3]. Support is costly financially. They are common in lowincome patient populations where improving the quality of care is a public health emergency, in order to reduce the cost of avoidable health care costs. According to some studies conducted in Europe, the prevalence of pressure ulcers varies from 0.5 to $9 \%$ depending on the populations at risk [3]. In 
Africa, we have not had any exploitable statistic in the literature on this condition. In Mali, there is also little data on the prevalence, mortality and morbidity of pressure ulcers in hospitals. According to the health pyramid, the Hôpital du Mali is a structure of last resort. This status gives it an establishment capable of receiving patients who are candidates for a long stay in hospital for chronic conditions that are often disabling. One of the hospital's missions is to improve the quality of care. The prevention and management of this complex preventable pathology are major challenges for healthcare staff. Yet, sometimes spectacular progress has been made in the prevention and management of pressure ulcers [4]. A study carried out in 2016 at the Hospital of Mali found a prevalence of pressure ulcers at $2.93 \%$ for all hospitalized patients and $5.1 \%$ for the emergency department, which had demonstrated the increase in nursing teams. 4 to 5 people per call and on-call and the involvement of caregivers in the care to improve the situation [4].

The objective of this study was to assess the prevalence of pressure ulcers in the emergency department at the Hôpital du Mali.

\section{Material and Method}

\subsection{Type and Scope of the Study}

This was a cross-sectional study from January 1 to December 31, 2020, conducted in the emergency reception service of the Hôpital du Mali in Bamako.

\subsection{Inclusion Criteria}

We included all admitted patients who stayed 3 days or more in the emergency department.

\subsection{Non-inclusion Criteria}

Patients who did not stay 3 days or more in the emergency department were not included in this study.

\subsection{Data Collection and Processing}

The data was collected on individual media developed for the purposes of the study.

These supports have been completed by the supervisor on duty. Social demographic and clinical parameters were analyzed for each patient. Data processing and entry was performed using SPSS software version 25.0.

\subsection{Ethical Consideration}

During this study we respected the principles of confidentiality of information by keeping the identities of patients secret and taking into account their informed consent.

\section{Results}

According to this study, we had registered 305 patients admitted to the emergency department.
Table 1. Distribution of pressure ulcer cases in the emergency department

\begin{tabular}{lll}
\hline Pressure ulcer & Effective & Percentage \\
\hline Yes & 15 & $4,9 \%$ \\
No & 290 & 95,1 \\
Total & 305 & $100 \%$ \\
\hline
\end{tabular}

We had found $4.9 \%$ developed pressure ulcers at the emergency level.

The sex ratio was 1.2. The mean age of our series was $52.49 \pm 14$ years old, with 21 years old as the minimum age and 87 years as the maximum age. Seventy percent $(70 \%)$ of the pressure ulcers were discovered at stage I. $2 / 15$ (13.33\%) of the pressure ulcers had come from the house and classified as stage IV, of which one case progressed to stage unclassified. Among the cases of pressure ulcer 1/15 (6.66\%) progressed to the unclassified stage.

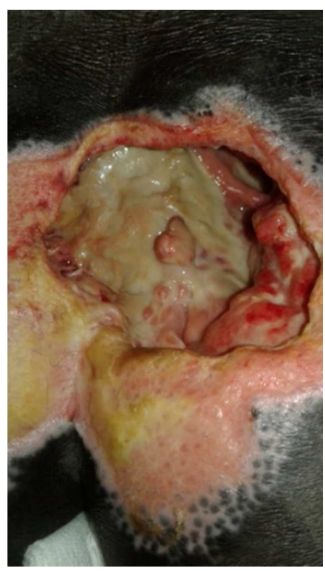

Figure 1. Photo of an unclassified pressure sore appearing at home (by phone at the Hôpital du Mali).

However, the pressure sore was managed using conventional dressing techniques by adding egg white during the healing phase.

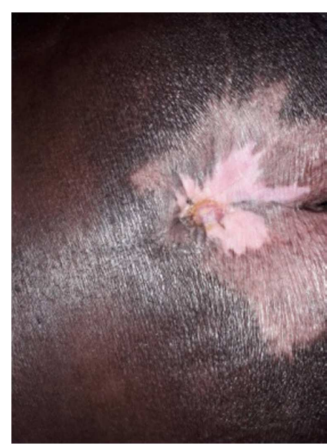

Figure 2. Photo of an unclassified pressure sore appearing at home completely healed after two months of treatment (taken by phone at the Hôpital du Mali).

Pressure ulcers located in the inter-gluteal folds were the most frequent with $80 \%$. We observed that $55 \%$ of pressure ulcers were developed in patients admitted for cervical and / or dorsolumbar trauma. Ninety-eight percent $(98.9 \%)$ of caregivers interviewed knew the risk factors for pressure ulcers.

Ninety-eight percent $(98.9 \%)$ of caregivers interviewed 
knew the risk factors for pressure ulcers.

\section{Discussion}

The objective of this study was to reassess the prevalence of pressure ulcers in the emergency reception service of the Hôpital du Mali. Aspects of the management and prevention of patient-related factors have not been addressed in this work.

Over one year, we found a prevalence of $4.9 \%$ of pressure ulcers. This prevalence slightly decreased between 2016 and 2019 in this service. In fact, Dembélé [4] found in 2016 a prevalence rate of $5.17 \%$ of pressure ulcers.

This decrease can be explained by the fact that the on-call teams have been increased and that we have involved carers in pressure ulcer prevention actions. Our results are comparable to those of Fromantin et al. Who found a prevalence of $5 \%$ in a pressure ulcer management unit in an oncology department [5] This clearly shows that pressure ulcers are a particularly common pathology all over the world. We found in this study a sex ratio of 1.2. Our results are comparable to those of SAMAKE, which found a sex ratio of 1.4 during a study on the grounds for admission of elderly people to intensive care at the CHU Gabriel TOURE [6].

We found an average age during this study of $52.49 \pm 14$ years, with 21 years as minimum and 87 years as maximum, this result is comparable to that of Dembele, [4] who finds 50 years as average age. On the other hand, Samake et al. found in their study an age ranges of 65 to 74 years with $63.3 \%$ [6]. This difference is explained by the reasons for hospitalization on the one hand and on the other hand by the nutritional status of the patients. We found that $70 \%$ of bedsores were discovered in stage I. According to Bernard this stage is equivalent to the appearance of blister, or more simply of blisters on the red zone. The blisters can be open or closed (the opening being done at the slightest local trauma), at this stage healing is easy and inexpensive, because the damage is not yet definitive [7-9].

These results show that the involvement of the family and the empowerment of nurses by care sector were effective for the detection and management of pressure ulcers, these results compared to those of Dembélé [4] who found $80 \%$ of the pressure ulcers discovered. At stage I, had progressed to stage II. At this stage, the skin is severely affected and there is necrotic type damage to muscle tissue. The skin is blackened, with some reddish colors, and becomes very dry. Clinically, this stage manifests as a local crater, so prevention is the best strategy, which consists of identifying risk factors, making regularly planned changes in position and maintaining skin hygiene $[1,5,10]$.

This is why the support of the family is essential for the regular change of position of the patients. Pressure ulcers in the buttocks more precisely in the inter-gluteal folds were the most frequent with $80 \%$. We noted that the situation has not changed at this level, because Dembélé [4] found $75 \%$ of pressure ulcers in the inter-gluteal space, this phenomenon is linked to the reasons for admission of patients who remained the same at the service level and posing a high risk of pressure ulcer development $[11,12]$. We also found $55 \%$ of bedsores were present in cervical and / or dorsolumbar trauma patients, these patients are always in dorsal decubitus positions thus facilitating skin abrasion with the bed.

During the study, $98.9 \%$ of the workers questioned knew the factors that cause pressure ulcers. This is explained by a good communication between the care teams and the families of the patients, because the involvement of the family is necessary for the regular change of position every 2 to 3 hours the sick person at risk of pressure ulcers. 'she is lying down or sitting down and this makes it possible to relieve the pressure at the level of the support points and to alternate the support zones [13-15].

Among the cases of pressure ulcer 1/15 (6.66\%) progressed to the unclassified stage. However, the pressure sore was managed using conventional dressing techniques by adding egg white during the healing phase. Chacon and Soban find that the evolution of pressure ulcers from one stage to another is linked on the one hand to the clinical and nutritional state of the patient and on the other hand to the quality of the technical management as as dressings and position changes $[16,17]$.

\section{Conclusion}

This study allowed us to understand that at the level of the emergency department of the Hôpital du Mali, the prevention of bedsores remains problematic, even if the rate of prevalence has decreased during the last four years, the bedsores make it difficult to take in charge of bedridden patients in the emergency department, thus, for their prevention there was an involvement of families to strengthen the changes in the position of patients, however the measures taken to slow down the evolution of pressure ulcers remains to be improved.

\section{Conflict of Interest}

With respect to this article there was no competing conflict of interest between the co-authors.

\section{References}

[1] Berlowitz D., (2014) Incidence and prevalence of pressure ulcers. In: Pressure Ulcers in the Aging Population. Springer; p. 19-26.

[2] National Agency for Accreditation and Assessment, Consensus Conference: Prevention and treatment of pressure ulcers in adults and the elderly. Georges-Pompidou European Hospital - Paris. 2001. https://www.hassante.fr/upload/docs/application/pdf/escarresdef_long.pdf, accessed September 12, 2020.

[3] Defloor T., Gobert M., Bouzegta N., Beeckman D., Vanderwee K., Van D. T., (2008) Study of the prevalence of pressure ulcers in Belgian hospitals-PUMap Project. 
[4] Dembélé B., Koné AZ, Sow, DS, Attaher O., Kone A., Doumbia N., Diarra B., Sidibe AT, Ouattara, Z., Diallo Y. L, Ouattara MH, Mody., Dakouo J., Sow FT, Maiga, BA, Camara FK, Sanogo A., Coulibaly CA, (2019), Survey on the prevention and management of bedsores at the hospital in Mali, Revue Malienne de Science et de Technologie serie B. Flight. 0 No 22.

[5] Fromantin I., Falcou MC., Baffie A., Petot C., Mazerat R., Jaouen C., (2011) Inception and validation of a pressure ulcer risk scale in oncology. J Wound Care. 20 (7): 328-334.

[6] Samaké B. M., Mangané SM, Togola M, Mangané M, Diani N, Diallo A. Reasons for admitting elderly people in intensive care to the Gabriel Toure University Hospital in BAMAKO. Mali Med. 2015; 30 (1).

[7] Fofana Y., Traore B., Faye O. and Al. Geriatric dermatoses in dermatological hospitalization in Bamako. Pan Afr Med J. 2016; 25 (206)

[8] Bernard, P., 2005. Complications of supine immobility. Prevention and management: bedsore. Annals of Dermatology and Venereology, 132, 7S11-7S15.

[9] Ceelen K. Aetiology of pressure ulcers A literature review. Part I of MSc-thesis, Eindhoven university of Technology, Faculty of Biomedical Engineering. 2003.

[10] Keller BPJA, Wille J, van Ramshorst B, Wvd C. Pressure ulcers in intensive care patients: a review of risks and prevention. Intensive Care Med. 2002; 28: 1379-1388.
[11] EPUA Panel. National Pressure Ulcer Advisory Panel. Prevention and treatment of pressure ulcers: guick reference guide Washington DC National Pressure Ulcer Advisory Panel; 2009.

[12] Benbow M. Guidelines for the prevention and treatment of pressure ulcers. Nurs Stand (through 2013) 2006; 20 (52): 42.

[13] Berlowitz D. Incidence and prevalence of pressure ulcers. In: Jini J, editor. Pressure ulcers in the aging population. New York: Springer; 2014. pp. 19-26.

[14] Bluestein D, Javaheri A. Pressure ulcers: prevention, evaluation, and management. Am Fam Physician. 2008; 78 (10).

[15] Macgregor L. International guidelines. Pressure ulcer prevention: prevalence and incidence in context. Consensus document. London: MEP Ltd.; 2009.

[16] Soban LM, Hempel S, Munjas BA, Miles J, Rubenstein LV. Preventing pressure ulcers in hospitals: a systematic review of nurse-focused quality improvement interventions. Jt Comm J Qual Patient Saf. 2011; 37 (6): 245.

[17] Chacon JMF, Blanes L, Hochman B, Ferreira LM. Prevalence of pressure ulcers among the elderly living in long-stay institutions in São Paulo. Sao Paulo Med J. 2009; 127 (4): 211-215. 